\title{
Etat d'urgence sanitaire pour faire face à l'épidémie de covid-19 en République Démocratique du Congo.
}

\author{
Brève évaluation du cadre juridique.
}

\author{
Par Symphorien KAPINGA K. NKASHAMA*
}

\section{Résumé}

La crise sanitaire due au Covid-19 a mis à rude épreuve le constitutionalisme et l'état de droit en République Démocratique du Congo comme dans beaucoup de pays au monde. Dans cette étude, l'auteur essaie de démontrer comment la gestion de la crise sanitaire par les autorités politiques congolaises a mis à nu l'incohérence des dispositions constitutionnelles relatives à l'état d'urgence et les lacunes de la législation sur cette matière. De même, l'intervention de l'Assemblée nationale et du Sénat dans un format réduit, sans quorum requis par la Constitution et les règlements intérieurs, pour proroger l'état d'urgence sanitaire, a révélé l'inadéquation des règles de fonctionnement de ces deux chambres du Parlement, aux exigences de la lutte contre de la pandémie du Covi-19. D'où, la nécessité pour le Parlement congolais de combler ce vide juridique en adoptant la loi portant modalités d'application de l'état d'urgence en tant que régime exceptionnel dérogatoire à l'ordre juridique établi et d'assouplir les règles de quorum en cas de circonstances exceptionnelles comme celles dues à la pandémie du Covid-19.

\begin{abstract}
The health crisis caused by covid-19 has severely tested constitutionalism and the rule of law in the Democratic Republic of Congo as in many countries around the world. In this study, the author tries to demonstrate how the management of the health crisis by the Congolese political authorities exposed the inconsistency of the constitutional provisions relating to the state of emergency and the gaps in the legislation on this matter. Similarly, the intervention of the National Assembly and the Senate in a reduced format, without a quorum required by the Constitution and internal regulations, to extend the state of health emergency, revealed the inadequacy of the operating rules of these two Houses of Parliament, to the demands of the fight against the covi-19 pandemic. Hence, the need for the Congolese Parliament to fill this legal void by adopting the law establishing modalities of application of the state of emergency as an exceptional regime derogating from the establi-
\end{abstract}

* Diplômé d'Etudes Supérieures en droit public (DES/DEA), Chef des travaux de Université de Mbujimayi (UM), et chercheur au Centre de Recherches et d'Etudes sur l'Etat de droit en Afrique (Creeda), E-mail : symphorienkapinga@gmail.com. 
shed legal order and to relax the quorum rules in the event of exceptional circumstances such as those due to the Covid-19 pandemic.

\section{Introduction}

C'est depuis décembre 2019 que le monde fait face à un virus très contagieux, identifié comme un nouveau coronavirus et dénommé Covid-19. Parti de la Chine ${ }^{1}$, ce virus a non seulement contaminé les populations d'Asie, d'Europe, d'Amérique, et d'Afrique, il a surtout décimé et fauché de nombreuses vies humaines. La République Démocratique du Congo (RDC) n’y a pas échappé. La situation épidémiologique à la date du 19 décembre 2020 fait état 15.494 cas confirmés, dont 370 décès et 13.447 personnes guéries ${ }^{2}$.

$\mathrm{Au}$ lendemain de l'annonce du premier cas diagnostiqué à Kinshasa au mois de mars 2020, le Président de la République, Félix Antoine Tshisekedi Tshilombo a, dans un message radiotélévisé diffusé le 18 mars 2020, annoncé les premières mesures prises par le Gouvernement pour limiter la propagation de la maladie et protéger la vie de la population ${ }^{3}$. Six jours après, devant l'accroissement quasi quotidien du nombre des personnes contaminées, le risque de la propagation de la maladie à l'échelle nationale étant avéré, le Président de la République a, après avoir consulté le Premier Ministre et les Présidents de deux chambres du Parlement, proclamé l'état d'urgence sanitaire sur l'ensemble du territoire national ${ }^{4}$. Des nouvelles mesures prises pour lutter contre cette pandémie ont restreint les droits humains et les libertés publiques ${ }^{5}$.

Et, si les effets du covid-19 ont eu un impact considérablement négatif sur la situation économique et sociale ${ }^{6}$, l'ordre juridique ainsi que le fonctionnement des institutions poli-

1 Sur l'évolution de la maladie dans le monde, voir https://www.ledevoir.com/documents/special/20-0 3_covid19-carte-dynamique/index.html; https://www.who.int/fr/emergencies/diseases/novel-corona virus-2019, consultés ce 19 décembre 2020.

2 Situation épidémiologique mise à jour le 07 avril 2020 moment où l'on clôturait la rédaction de ce papier disponible sur https://www.stopcoronavirusrdc.info/ consulté le 08 avril 2020.

3 Felix Antoine Tshisekedi Tshilombo, Message à la nation relatif à la pandémie du coronavirus, covid-19, Kinshasa, Kinshasa, Mercredi 18 Mars 2020, disponible sur https://www.presidence.cd/uplo ads/files/Message\%20COVID-19\%20_\%20Mars\%202020.pdf.pdf.

4 Felix Antoine Tshisekedi Tshilombo, Message à la nation relatif à la pandémie du coronavirus, covid-19, Kinshasa, Mardi 24 Mars 2020, disponible sur https://fr.africanews.com/2020/03/24/corona virus-republique-democratique-du-congo-message-a-la-nation-de-sem-felix-antoine-tshisekedi-tshil ombo-president-de-la-republique-chef-de-letat-relatif-a-la-pandemie-du-coronavirus-covid-19//.

5 Ordonnance n²0/014 du 24 mars 2020 portant proclamation de l'Etat d'urgence sanitaire pour faire face à l'épidémie du covid-19, JORDC, Numéro spécial, 16 avril 2020, pp.5 - 10; Arrêté Ministériel n`25 du 25 mars 2020 portant modalités pratiques d'exécution des mesures de limitation de la propagation de la pandémie du Covid-19, disponible sur https://www.juridocs.org/publications/read/arr ete-ministeriel-portant-modalites-pratiques-dexecution-des-mesures-de-limitation-de-la-propagation -de-la-pandemie-covid-19.

6 https://www.undp.org > UNDP-rba-Covid-RDC-DRC_2020, consulté ce 19 décembre 2020. 
tiques $^{7} l^{1}$ 'ont également été. L'état d'urgence sanitaire ainsi proclamé, pour la première fois depuis l'entrée en vigueur de la Constitution du 18 février 2006, a révélé les caractères lacunaires et incohérents du régime juridique en la matière, en mettant ainsi à nu l'inadéquation du cadre fonctionnel des deux chambres du Parlement pour y faire face. Cette étude entend en faire la démonstration.

\section{La crise sanitaire comme événement grave justifiant la proclamation de l'état d'urgence}

L'état d'urgence est un régime exceptionnel dérogatoire au droit commun qui régit l'organisation des pouvoirs publics et garantit les droits et libertés fondamentales des citoyens. C'est la raison pour laquelle sa proclamation est conditionnée par l'existence des circonstances graves qui menacent les intérêts stratégiques de la République. C'est ce qui ressort de la disposition de l'article 85, alinéa 1 de la Constitution qui dispose: « lorsque des circonstances graves menacent, d'une manière immédiate, l'indépendance ou l'intégrité du territoire national ou qu'elles provoquent l'interruption du fonctionnement régulier des institutions de la République le Président de la République proclame l'état d'urgence ou l'état de siège après concertation avec le Premier ministre et les Présidents des deux Chambres conformément aux articles 144 et 145 de la présente Constitution» ${ }^{8}$.

La Constitution n'indique pas clairement les événements graves qui peuvent donner lieu à la proclamation de l'état d'urgence ou de l'état siège. Elle ne distingue pas non plus la crise sanitaire de la crise sécuritaire. Il sied de constater que depuis l'entrée en vigueur de la Constitution, la loi qui devait fixer les modalités d'application de l'état d'urgence n'a pas encore été adoptée jusqu'à ce jour prévue par l'alinéa 3 de l'article 85 de la Constitution; ce qui constitue une lacune grave de la législation congolaise en cette matière.

Si selon la doctrine, l'état de siège suppose une guerre ouverte contre une puissance étrangère ou un mouvement rebelle, qui mettrait en péril l'indépendance ou l'intégrité du territoire et à laquelle le Gouvernement devrait faire face, l'état d'urgence implique, quant à lui, tout événement autre que la guerre mais qui menacerait la vie de la Nation ou le fonctionnement régulier des institutions ${ }^{9}$.

En l'espèce, l'épidémie de coronavirus (Covid-19) qui sévissait au pays, au regard de ses effets dévastateurs, a été considéré comme un événement grave qui menaçait, de manière immédiate, la vie de la Nation et le fonctionnement régulier des institutions de la République. Cela ressort clairement de l'Ordonnance n²0/014 du 24 mars 2020 proclamant cet état d'urgence sanitaire. On y lit dans son exposé des motifs le passage suivant :

7 Lire aussi à ce sujet Kambale Kambale Pascal, Impact de la crise de covid-19 sur le processus démocratique en RDC, disponible sur http://congoresearchgroup.org/impact-de-la-crise-de-covid-19-s ur-le-processus-democratique-en-rdc/?lang=fr.

8 Article 85 alinéa 1 de la Constitution du 18 février 2006 telle que modifiée et complétée à ce jour, JORDC, Numéro spécial, Kinshasa, 6 février 2011.

9 Alphonse Ntumba Luaba Luтu, Droit constitutionnel général, Kinshasa, 2007, p. 318. 
Considérant que la propagation actuelle, inédite, imprévisible et rapide du Coronavirus, COVID-19,... laquelle propagation a provoqué l'interruption du fonctionnement des institutions de la République (... $)^{10}$.

La question à laquelle il va falloir répondre est celle de savoir si le Président de la République a la qualité d'apprécier seul le caractère menaçant de la situation nécessitant la proclamation de cet état d'exception et quel est l'étendue de ses pouvoirs dans cette situation.

\section{Le Président de la République comme autorité d'appréciation et de proclamation de l'état d'urgence}

Il ressort des dispositions des articles 85 alinéa 1 et 144 alinéa 1 de la Constitution du 18 février 2006 telle que modifiée à ces jours que le Président de la République est l'autorité compétente instituée pour proclamer et déclarer l'état d'urgence ${ }^{11}$. L'alinéa 1 de l'article 85 sus-évoqué est libellé comme suit :

Lorsque des circonstances graves menacent (...), le Président de la République proclame l'état d'urgence ou l'état de siège après concertation avec le Premier ministre et les Présidents des deux Chambres conformément aux articles 144 et 145 de la présente Constitution.

Comme on peut le remarquer, la proclamation de l'état d'urgence comme l'état de siège est tributaire d'un préalable, à savoir la concertation avec le Premier Ministre et les Président de deux chambres du Parlement. Par la concertation, le Président de la République n'est pas la seule autorité à apprécier la circonstance grave menaçant immédiatement l'indépendance ou l'intégrité du territoire national ainsi que provoquant l'interruption du fonctionnement régulier des institutions. Le Premier Ministre et les Présidents de l'Assemblée nationale et du Sénat y sont associés, même si la Constitution ne précise pas l'acte qui devrait constate la preuve de la concertation. Est-ce par un communiqué ou un procès-verbal ?

Dans le cas sous-examen, c'est à la suite de cette formalité que cet état d'urgence est proclamé. Le Président de la République avait, après avoir rencontré le Premier et les Présidents de deux chambres du Parlement, pris l'Ordonnance n²0/014, en date du 24 mars 2020 pour faire face à la pandémie du Covid-19. Il en a informé la nation par un message radiotélévisé, diffusé sur les ondes de la Radio Télévision Nationale Congolaise (RTNC). Mais au lendemain de l'annonce de cette ordonnance et des mesures qu'elle portait pour faire face à la situation sanitaire créée par le covid-19, une vive polémique a éclaté sur la question de savoir si le Chef de l'Etat devrait requérir ou non l'autorisation préalable du Parlement avant de proclamer l'état d'urgence sanitaire. C'est pour contribuer à ce débat qu'il est important d'examiner le rôle du Parlement en cette matière.

10 Exposé des motifs de l'ordonnance n²0/014 du 24 mars 2020, 4eme considérant, note 5.

11 Jacques Djoli Eseng'Ekeli, Droit constitutionnel. L'expérience congolaise(RDC), Paris, 2013, p.205. 


\section{B. LE ROLE DU PARLEMENT DANS LA SITUATION DE L'ÉTAT D'URGENCE}

Si le pouvoir du Parlement de proroger ou de mettre fin à l'état d'urgence est clairement défini dans la Constitution, celui d'autoriser préalablement la proclamation de l'état par le Président a suscité de la controverse dans l'opinion publique.

\section{Le Parlement et l'autorisation préalable à la proclamation de l'état d'urgence}

Pour revenir sur cette controverse en rapport au rôle du Parlement, il sied de rappeler qu'elle a été alimentée par le Président du Sénat qui, recourant à l'article 119 de la Constitution, considérait que le Congrès était nécessaire pour autoriser la proclamation de l'état d'urgence et que le Président n'a pas respecté la Constitution quant à ce ${ }^{12}$. Cet article 119 dispose : « les deux Chambres se réunissent en Congrès pour les cas suivants : (...) 2. L'autorisation de la proclamation de l'état d'urgence ou de l'état de siège et de la déclaration de guerre, conformément aux articles 85 et 86 de la présente Constitution (...) ».

La rédaction de cette disposition nous parait peu heureuse en son alinéa 2. Ses prescrits nous semblent manifestement contradictoires à ceux de l'article 85 auquel elle se réfère pourtant; ce qui prête à confusion sur le régime juridique applicable à l'état d'urgence. C'est ce qu'avait constaté en son temps la Cour suprême de justice, toutes sections réunies lorsque, se prononçant sur la constitutionnalité du règlement intérieur du Congrès, elle avait considéré à juste titre que l'article 119 de la Constitution s'éloignait des prescrits de l'article 85 qui n'exigent pas l'autorisation du Parlement pour la proclamation de l'état d'urgence $^{13}$.

Pour sa part, la Cour constitutionnelle a un avis tout à fait contraire à ce sujet en soutenant une position qui n'est pas à l'abri des critiques. En effet, dans son arrêt en appréciation de la conformité à la Constitution de l'Ordonnance n²0/014 du 24 mars 2020 portant proclamation de l'état d'urgence sanitaire pour faire face à l'épidémie de Covid-19, ses membres soutiennent :

Le constituant n'a pas déterminé la forme de ces circonstances, lesquelles relèvent de la souveraine appréciation du Président de la République qui peut opter pour la proclamation de l'état d'urgence ou l'état de siège après concertation avec le Premier Ministre et les deux chambres du Parlement ou soit il peut, le cas échéant et selon les circonstances, saisir le Congrès en application de l'article 119 point 2 de la Constitution $^{14}$.

12 Pour les détails sur les propos du Président du Sénat Alexis Tambwe Mwamba, voir https://congoa ctu.net/rdc-felix-tshisekedi-navait-pas-consulte-le-congres-pour-decreter-letat-durgence-thambwemuamba; https://7sur7.cd/2020/04/08/rdc-covid-19-un-congres-sera-bientot-convoque-pour-statuer -sur-les-modalites?fbclid=IwAR2btHYzaXVs41sDr1ArFXolPW_3oK5smQlHALvoiEfJIMVew8J $1 \mathrm{CjNtVt4.}$

13 CSJ, RCons.063/TSR, arrêt du 30 novembre 2007, inédit.

14 CC, R.Const. 1200, Arrêt du 13 avril 2020, JORDC, Numéro spécial, 16 avril 2020, p.18. 
Au regard de cette position de la Cour que nous ne partageons pas du reste, le Président de la République aurait deux options : soit se concerter avec les personnalités identifiées, soit requérir l'autorisation du Congrès. Ainsi, pour les membres de la Cour, en choisissant la concertation en lieu et place de l'autorisation du Congrès, le Président de la République n'avait en rien violé la Constitution ${ }^{15}$ comme le lui reprochait le Président du Sénat.

\section{Le Parlement et la prorogation de l'état d'urgence}

Qu'à cela ne tienne, le Parlement congolais a un rôle à jouer lorsque l'état d'urgence est proclamé. Etant donné que cette proclamation est faite conformément aux dispositions des articles 144 et 145 de la Constitution, le rôle du Parlement y est défini de manière plus ou moins claire. En l'espèce, aux termes des alinéas 1 et 2 de l'article 144 de la Constitution,

L'Assemblée nationale et le Sénat se réunissent alors de plein droit. S'ils ne sont pas en session, une session extraordinaire est convoquée à cet effet conformément à l'article 116 de la présente Constitution. La clôture des sessions ordinaires ou extraordinaires est de droit retardée pour permettre, le cas échéant, l'application des dispositions de l'alinéa précédent ${ }^{16}$.

La convocation de la session, extraordinaire soit-elle, et le retardement de sa clôture en cas d'état d'urgence démontrent à suffisance que le Parlement est appelé à jouer un rôle important dans ce régime exceptionnel, même si celui-ci n'est pas clairement défini. Dans la mesure où l'état d'urgence qui peut être proclamé sur tout ou partie du territoire l'est pour une durée de trente jours ${ }^{17}$, l'ordonnance l'ayant décrété cesse de plein droit de produire ses effets après cette expiration du délai, à moins que l'Assemblée nationale et le Sénat, saisis par le Président de la République sur décision du Conseil de Ministres, n'en aient autorisé la prorogation pour des périodes successives de quinze jours ${ }^{18}$.

Ainsi, à l'expiration de chaque délai de 30 jours de l'état d'urgence et face à la montée toujours croissance du nombre de cas contamination au covid-19, le Président de la République a dû solliciter et obtenir de l'Assemblée nationale et du Sénat, la prorogation par voie législative de l'état d'urgence sanitaire pour des périodes de 15 jours pendant 4 mois. L'objectif visé était de permettre au Gouvernement de poursuivre les mesures indispensables pour limiter la propagation de la pandémie liée au covid-19 19 .

Autant que l'Assemblée nationale et le Sénat ont le pouvoir d'autoriser la prorogation de l'état d'urgence pour la période indiquée, autant ils peuvent y mettre fin. Le constituant

15 CC, R.const.1200, Arrêt du 13 avril 2020, note 14.

16 Article 144, al.1 et 2 de la Constitution du 18 février 2006, note 8.

17 Article 144, al.4 de la Constitution du 18 février 2006, note 8.

18 Article 144, al.5 de la Constitution du 18 février 2006, note 8.

19 https://www.radiookapi.net/2020/04/24/actualite/politique/covid-19-le-parlement-de-la-rdc-prorog e-letat-durgence-de-15-jours. 
dispose, à cet effet, que l'Assemblée nationale et le Sénat peuvent, par une loi, mettre fin à tout moment à l'état d'urgence $(. ..) »^{20}$.

Comme on peut le constater, le cadre d'intervention du Parlement congolais afin de proroger ou de mettre fin à l'état d'urgence sanitaire s'est avéré inadéquat. Cette inadéquation du cadre fonctionnel de deux Chambres s'est manifestée dès la déclaration des premiers cas de covid-19 dans la ville de Kinshasa. En effet, les bureaux de deux chambres du Parlement avaient suspendu leurs sessions de mars afin d'éviter le risque de contamination des députés et sénateurs ainsi que des membres du personnel de deux chambres. Pourtant, l'Ordonnance portant proclamation de l'état d'urgence sanitaire pour faire face à l'épidémie du covid-19 n'avait pas interdit le fonctionnement des institutions étatiques. Au contraire, il était demandé de prendre de mesures nécessaires pour éviter la propagation de la maladie. C'est qui ressort de l'alinéa 1 de l'article précité libellé comme suit :

Les chefs et responsables de toutes les institutions, tant nationales que provinciales, sont tenus de prendre des dispositions sanitaires idoines pour mettre leurs membres et personnels ainsi que l'ensemble de la population à l'abri de toute contamination ou toute propagation de la contamination au coronavirus, COVID-1921.

Mais en pratique, la difficulté s'est avérée réelle : comment réunir des nombreux députés ou sénateurs en plénières de l'Assemblée nationale ou du Sénat tout en respectant les règles de distanciations sociales exigées dans la lutte contre la propagation du Covid-19? Tel était le grand défi que les bureaux de deux chambres du Parlement devaient relever au regard de l'exigence du quorum pour siéger valablement.

En effet, aux termes de l'article 118 alinéa $1^{\text {er }}$ de la Constitution repris comme tels dans les règlements intérieurs de deux chambres ${ }^{22}$, 1 'Assemblée nationale et le Sénat ne peuvent siéger valablement qu'à la majorité absolue des membres qui les composent. Or, suite à la pandémie du Covid-19, aux exigences des gestes et des règles de distanciations sociale qui ont été imposées à tous, même aux institutions publiques pour éviter des contaminations au covid-19, il s'est avéré difficile de réunir plus de 250 députés nationaux pour l'Assemblée nationale.

C'est ainsi que pendant l'état d'urgence sanitaire les bureaux des deux chambres parlementaires ont dû recourir aux plénières en format réduit, sans considération de quorum requis pour siéger afin d'adopter les lois autorisant les multiples prorogations de ce régime d'exception. Par exemple, la plénière de l'Assemblée nationale ne comptait à peine 66 dé-

20 Article 144, al.6 de la Constitution du 18 février 2006, note 8.

21 Article 5 de l'Ordonnance n²0/014 du 24 mars 2020, note 5.

22 Article 69 du Règlement intérieur de l'Assemblée nationale et 79 du Règlement intérieur du Sénat. 
putés nationaux au moment du vote de la Loi de prorogation de l'état d'urgence le 23 avril et le 7 mai $2020^{23}$.

Ce qui constitue, à notre avis, une violation de la Constitution et des règlements intérieurs de l'Assemblée nationale et du Sénat. C'est un élément révélateur de cette inadéquation du cadre juridique régissant le fonctionnement de ces deux chambres parlementaires. Il y a là nécessité de l'amender afin de l'adapter aux circonstances exceptionnelles comme celles imposées à la suite de cette crise sanitaire due au Covid-19.

\section{Conclusion}

Les effets du covid-19 n'ont épargné aucun domaine de la vie sociale en RDC comme dans tous les pays au monde. Mais, s'ils sont plus ressentis dans les domaines économique et social, le domaine juridique ou l'état de droit en a aussi souffert. La brève évaluation du cadre juridique de l'état d'urgence sanitaire décrété en RDC depuis le mois de mars 2020 pour faire face à l'épidémie du Covid-19 que nous venons de présenter a montré les lacunes de la législation congolaise qui ne dispose pas jusqu'à ce jour de la loi portant modalités d'application de l'état d'urgence en tant que régime exceptionnel dérogatoire à l'ordre juridique établi, institué par le constituant pour permettre au Gouvernement de faire face à certaines situations, en dehors de la guerre, mais qui sont susceptibles d'interrompre le fonctionnement régulier des institutions ou de menacer la vie de la nation comme le covid-19.

C'est ce qui explique en grande partie les hésitations des acteurs politiques et les controverses sur la validité de tel ou tel acte pris par telle autorité. D'où, la nécessité pour le Parlement de combler ce vide juridique en adoptant le plutôt la loi portant modalités d'application de l'état d'urgence prévue par la Constitution ${ }^{24}$ et qui fait défaut jusqu'à ce jour.

Par ailleurs, l'intervention de l'Assemblée nationale et le sénat dans un format réduit sans respect du quorum requis, afin d'autoriser par voie législative la prorogation de l'état d'urgence sanitaire, nécessaire pour la poursuite de la lutte contre l'épidémie due au COVID-19 ou de déclarer la fin de ce régime exceptionnel, a révélé l'inadéquation du cadre juridique du fonctionnement de ces chambres parlementaires et le besoin d'assouplir les règles relatives au quorum afin de les adapter aux exigences des situations d'urgences comme celles imposées par la maladie due au covid-19.

Ceci implique en définitive l'amendement non seulement de l'alinéa 1 de l'article 118 de la Constitution mais aussi des règlements intérieurs de ces deux chambres du Parlement afin de prévoir la possibilité de la tenue des plénières en format réduit ou par vidéo-conférence en cas des situations exceptionnelles comme celle liée à crise sanitaire due au covid-19.

23 Pour plus des détails, voir https://www.rtbf.be/info/monde/detail_coronavirus-en-rdc-le-parlementvote-la-prorogation-de-15-jours-de-l-etat-d-urgence?id=10488434; https://www.radiookapi.net/20 20/05/07/actualite/politique/rdc-le-parlement-proroge-letat-durgence-sanitaire.

24 Article 85 alinéa 3 de la Constitution, note 8 . 


\section{Bibliographie}

\section{Textes juridiques}

Constitution du 18 février 2006 telle que modifiée et complétée à ce jour, JORDC, Numéro spécial, Kinshasa, 6 février 2011.

Ordonnance n ${ }^{\circ} 20 / 014$ du 24 mars 2020 portant proclamation de l'Etat d'urgence sanitaire pour faire face à l'épidémie du covid-19, JORDC, Numéro spécial, 16 avril 2020, pp.5 - 10.

Arrêté Ministériel n²5 du 25 mars 2020 portant modalités pratiques d'exécution des mesures de limitation de la propagation de la pandémie du Covid-19, disponible sur -https://www.juridocs.org/pub lications/read/arrete-ministeriel-portant-modalites-pratiques-dexecution-des-mesures-de-limitation -de-la-propagation-de-la-pandemie-covid-19.

Règlement intérieur de l'Assemblée nationale, Kinshasa, 2019, inédit.

Règlement intérieur du Sénat, Kinshasa, 2019, inédit.

\section{Doctrine}

Alphonse Ntumba Luaba Lumu, Droit constitutionnel général, Kinshasa, 2007.

Felix Antoine Tshisekedi Tshilombo, Message à la nation relatif à la pandémie du coronavirus, covid-19, Kinshasa, Kinshasa, Mercredi 18 Mars 2020.

Felix Antoine Tshisekedi Tshilombo, Message à la nation relatif à la pandémie du coronavirus, covid-19, Kinshasa, Mardi 24 Mars 2020.

Jacques Djoli Eseng'Ekeli, Droit constitutionnel. L'expérience congolaise(RDC), Paris, 2013, p.205.

Kambale Kambale Pascal, Impact de la crise de covid-19 sur le processus démocratique en RDC, disponible sur http://congoresearchgroup.org/impact-de-la-crise-de-covid-19-sur-le-processus-democr atique-en-rdc/?lang=fr.

\section{Jurisprudence}

Cour Constitutionnelle, R.Const. 1200, Arrêt du 13 avril 2020, JORDC, Numéro spécial, 16 avril 2020, p.18.

Cour suprême de justice (CSJ), R.Cons.063/TSR, Arrêt du 30 novembre 2007, inédit.

\section{Sites internet}

https://congoactu.net/rdc-felix-tshisekedi-navait-pas-consulte-le-congres-pour-decreter-letat-durgence-t hambwe-muamba.

https://7sur7.cd/2020/04/08/rdc-covid-19-un-congres-sera-bientot-convoque-pour-statuer-sur-lesmodal ites?fbclid=IwAR2btHYzaXVs41sDr1ArFXolPW_3oK5smQ1HALvoiEfJIMVew8J1CjNtVt4.

https://www.rtbf.be/info/monde/detail_coronavirus-en-rdc-le-parlement-vote-la-prorogation-de-15-jour s-de-1-etat-d-urgence?id=10488434. 
https://www.stopcoronavirusrdc.info/

https://www.undp.org > UNDP-rba-Covid-RDC-DRC_2020, consulté ce 19 décembre 2020.

https://www.who.int/fr/emergencies/diseases/novel-coronavirus-2019

https://www.radiookapi.net/2020/05/07/actualite/politique/rdc-le-parlement-proroge-letat-durgence-san itaire.

https://www.ledevoir.com/documents/special/20-03_covid19-carte-dynamique/index.html.

https://fr.africanews.com/2020/03/24/coronavirus-republique-democratique-du-congo-message-a-la-nat ion-de-sem-felix-antoine-tshisekedi-tshilombo-president-de-la-republique-chef-de-letat-relatif-a-la -pandemie-du-coronavirus-covid-19//.

https://www.presidence.cd/uploads/files/Message\%20COVID-19\%20_\%20Mars\%202020.pdf.pdf.

https://www.radiookapi.net/2020/04/24/actualite/politique/covid-19-le-parlement-de-la-rdc-proroge-let at-durgence-de-15-jours. 